ARTICLE

\title{
Twisted-Planar-Twisted expanded porphyrinoid dimer as a rudimentary reaction-based methanol indicator
}

Qizhao Li ${ }^{1}$, Chengjie Li ${ }^{1}$, Glib Baryshnikov (1) ${ }^{2}$, Yubin Ding ${ }^{1}$, Chengxi Zhao ${ }^{1}$, Tingting Gu ${ }^{3}$, Feng Sha ${ }^{1}$, Xu Liang $^{3}$,

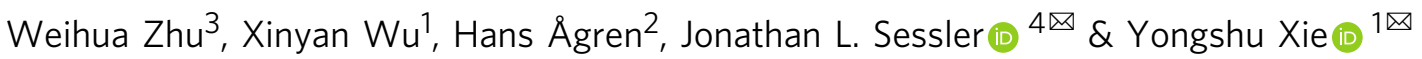

Directly linked porphyrin dimers have attracted considerable attention because of their intriguing electronic features. Most emphasis has been placed on either dimers with large dihedral angles between the constituent planar monomeric subunits or those with overall planarity, referred to as "Planar-Twisted-Planar" and "Planar-Planar-Planar", respectively. Herein, we report a "Twisted-Planar-Twisted" framework, the hexaphyrin dimer D that exists in a trans configuration. Treatment of $\mathbf{D}$ with $\mathrm{MeOH}$ affords two isomeric dimers, MD1 and MD2, both of which incorporate a methoxy moiety and exist in cis orientations with respect to the tethering linkage. The methanol-promoted conversion is accompanied by a readily discernible color change from green to brown and is not induced to an appreciable level by other alcohols. Dimer $\mathbf{D}$ thus acts as a rudimentary, albeit highly selective, reaction-based methanol indicator. This work provides a promising approach for constructing reaction-based chemosensors using porphyrinoid dimers of nonplanar subunits with biased reactivity.

\footnotetext{
${ }^{1}$ Key Laboratory for Advanced Materials and Joint International Research Laboratory of Precision Chemistry and Molecular Engineering, Feringa Nobel Prize Scientist Joint Research Center, Frontiers Science Center for Materiobiology and Dynamic Chemistry, School of Chemistry and Molecular Engineering, East China University of Science \& Technology, 130 Meilong Road, 200237 Shanghai, China. ${ }^{2}$ School of Biotechnology, KTH Royal Institute of Technology, SE10691 Stockholm, Sweden. ${ }^{3}$ School of Chemistry and Chemical Engineering, Jiangsu University, 212013 Zhenjiang, China. ${ }^{4}$ Department of Chemistry, The University of Texas at Austin, Austin, TX 78712-1224, USA. ${ }^{\star}$ email: sessler@cm.utexas.edu; yshxie@ecust.edu.cn
} 
aturally occurring tetrapyrrolic pigments, including porphyrin (Fig. 1a), play essential roles in both aquatic and terrestrial life, while various synthetic analogs have seen extensive application in a wide range of application areas, including in materials and medicinal chemistry ${ }^{1,2}$. This importance has provided an incentive to create porphyrin analogs, i.e., porphyrinoids, including expanded porphyrins, contracted porphyrins, core-modified porphyrins and isomeric porphyrins ${ }^{3,4}$.
The first member of this ever-increasing family, the pentapyrrolic macrocycle sapphyrin ${ }^{5}$, was disclosed by Woodward in 1966 as being an unexpected reaction by-product. Since then, numerous expanded porphyrins have been prepared and studied in light of their flexible conformations, rich coordination chemistry, unique redox activity, and unusual chemical reactivity ${ }^{6-8}$. Often these features have no parallel in the chemistry of simple porphyrins. Early on, for instance, it was found that under certain conditions

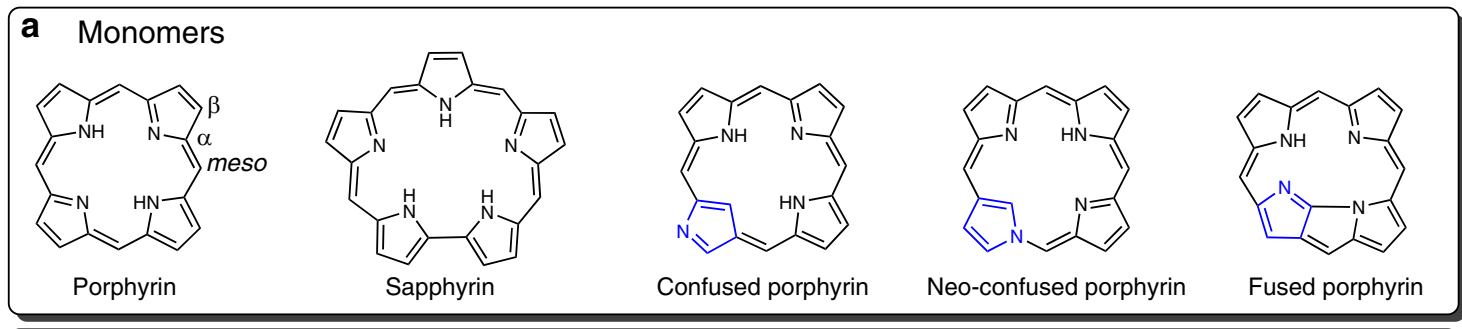

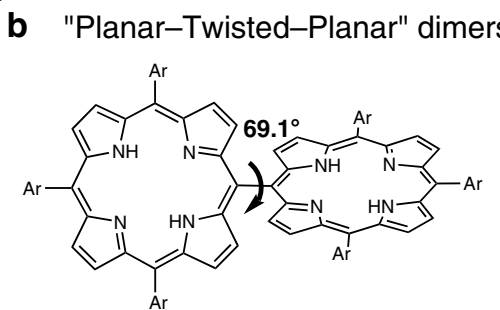

Porphyrin dimer (meso-meso)<smiles></smiles>

Confused porphyrin dimer $(\alpha-\alpha)$

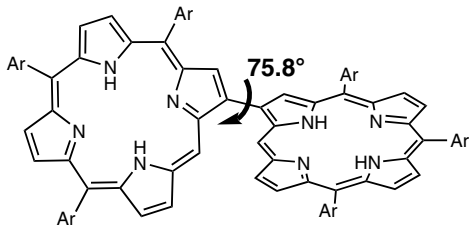

Porphyrin dimer $(\beta-\beta)$

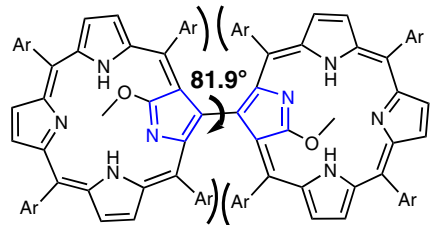

Confused porphyrin dimer $(\beta-\beta)$

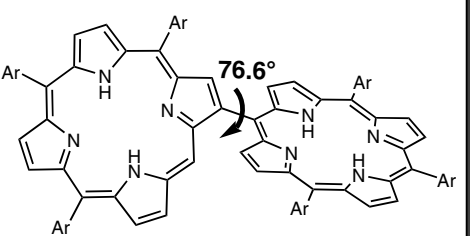

Porphyrin dimer ( $\beta$-meso)

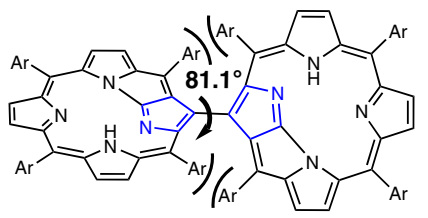

Fused porphyrin dimer $(\beta-\beta)$

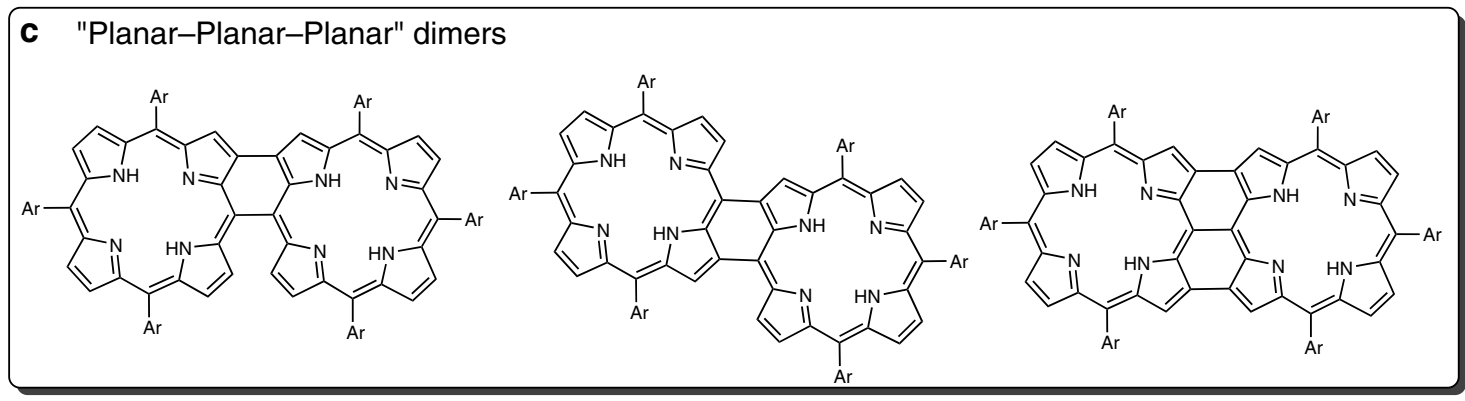

d "Twisted-Planar-Twisted" dimers (this work)

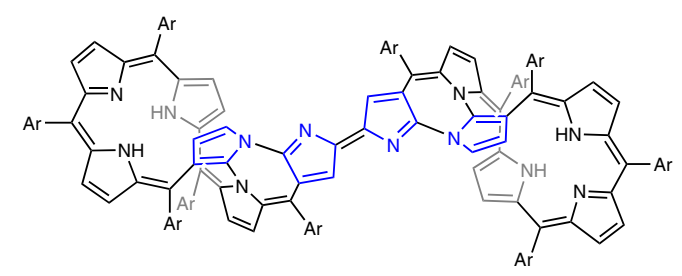

Neo-fused hexaphyrin dimer $\mathbf{D}(\alpha-\alpha)$

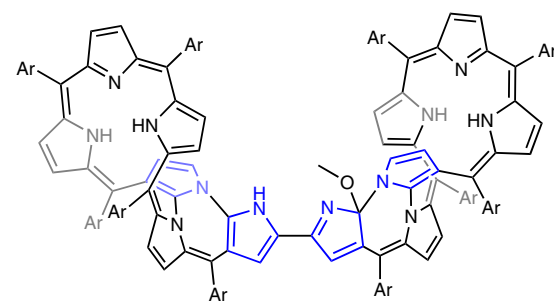

Neo-fused hexaphyrin dimer MD1 $(\alpha-\alpha)$

Fig. 1 Chemical structures of porphyrin, porphyrin analogs, and their dimers. a Porphyrin and related monomeric macrocycles; $\mathbf{b}$ examples of "Planar-Twisted-Planar" dimers: the two planar porphyrin/porphyrinoid units are linked in a highly twisted manner; c examples of "Planar-Planar-Planar" dimers: the two planar porphyrin units are linked by means of direct fusion; $\mathbf{d}$ "Twisted-Planar-Twisted" dimer $\mathbf{D}$ reported in this work: Note that the twisted hexaphyrin units are linked through a coplanar bipyrrolic linkage. Ar represents aromatic substituents. The blue-colored pyrrolic units represent confused pyrroles, which are linked with adjacent pyrroles or meso-carbons through $\mathrm{C}_{\alpha}-\mathrm{C}_{\beta}$ or $\mathrm{N}-\mathrm{C}_{\beta}$ atoms. 


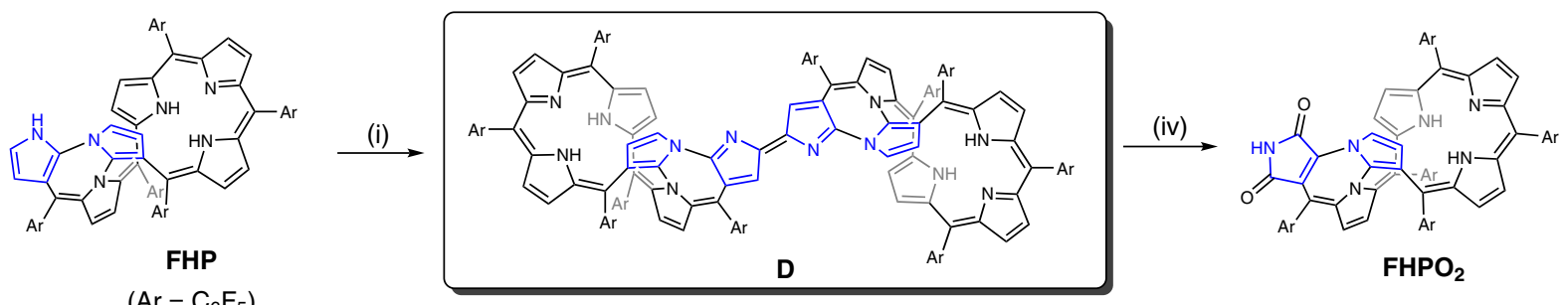

(ii), (iii)
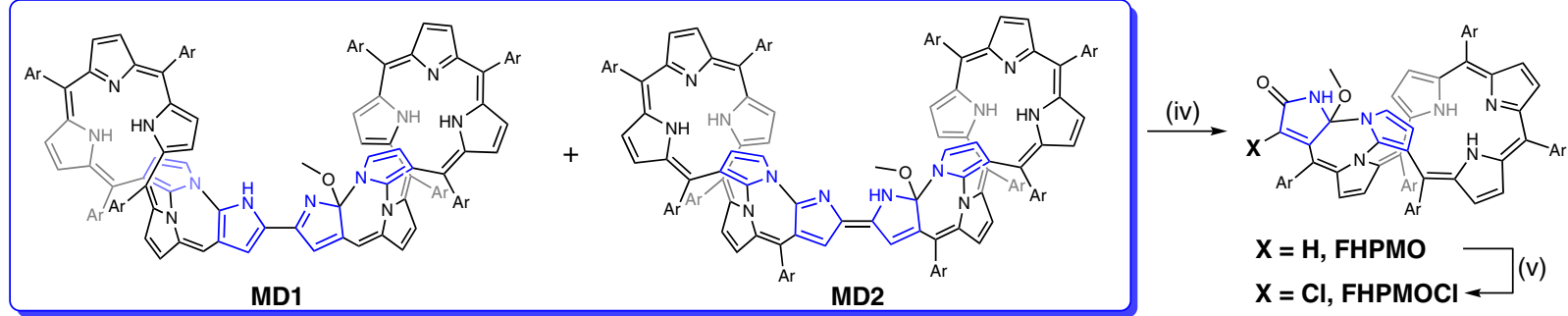

Fig. 2 Syntheses of dimers and monomers considered in this study. Conditions: (i) p-chloranil, $\mathrm{CHCl}_{3}$, reflux; (ii) $\mathrm{Cs}_{2} \mathrm{CO}_{3}, \mathrm{THF} / \mathrm{MeOH}$; (iii) TFA, THF/ $\mathrm{MeOH}$; (iv) $\mathrm{CF}_{3} \mathrm{COOAg}, \mathrm{CH}_{2} \mathrm{Cl}_{2} / \mathrm{MeOH}$; (v) $\mathrm{FeCl}_{3}, \mathrm{CHCl}_{3} / \mathrm{MeOH}$, reflux. The blue-colored pyrrolic units represent confused pyrroles that are linked with adjacent pyrroles or meso-carbons through $\mathrm{C}_{\alpha}-\mathrm{C}_{\beta}$ or $\mathrm{N}-\mathrm{C}_{\beta}$ atoms.

sapphyrin would undergo nucleophilic attack by methanol to produce a formally non-aromatic derivative ${ }^{9}$. Similar chemistry has been occasionally observed in the case of other porphyrinoid systems ${ }^{10-14}$. However, systematic efforts to exploit this reaction in the context of chemical sensing ("chemosensing") are to our knowledge unknown. Here we report a "Twisted-Planar-Twisted" dimeric expanded porphyrin system (D; Fig. 1) that reacts with methanol selectively and functions as a rudimentary, albeit selective, methanol chemosensor (formally a reaction-based indicator).

In recent years, considerable effort has been devoted to extending the chemistry of porphyrin analogs beyond the limits of simple macrocyclic systems to encompass, inter alia, two-/ three-dimensional structures ${ }^{15,16}$, linear porphyrin tapes ${ }^{17,18}$, and annular nano-rings ${ }^{19,20}$. Directly linked porphyrin dimers have received particular attention within the context of this general paradigm because of their interesting optical and electrochemical features $^{21,22}$. Confused porphyrins ${ }^{22,23}$ (containing a pyrrole unit linked at the $\alpha, \beta^{\prime}$ positions) have also been used for constructing such dimers. In this case, the unoccupied $\alpha$ position may also be exploited to link the subunits in addition to the $\beta$ - and mesopositions that normally serve as inter-porphyrinoid linkage sites (Fig. 1b) ${ }^{10,24}$, reflecting the inherent biased (and relatively high) reactivity of the a-pyrrolic positions. However, the directly linked bipyrroles that result from dimerization are usually severely twisted (reflected in large dihedral angles), presumably as the result of steric hindrance between the planar monomeric subunits. This results in "Planar-Twisted-Planar" dimers (Fig. 1b) and little electronic communication between the individual macrocyclic components. To address this limitation, post-linkage reactions have been used to fuse the two porphyrin units to give "Planar-Planar-Planar" porphyrin dimers 25,26 (Fig. 1c). Less well explored, and all but unknown, are porphyrinoid dimers with an overall "Twisted-Planar-Twisted" structure (Fig. 1d). Although a number of expanded porphyrins with twisted conformations (e.g., "figure-of-eight" structures) are known, they usually do not exhibit biased reactivity and are not readily dimerized in high yield ${ }^{27,28}$. However, we felt that if the confusion- and expansionbased approaches to porphyrin analog generation were combined, it might prove possible to obtain such dimers. Recently, we reported a neo-fused hexaphyrin FHP, a 5,5,5,7-tetracyclic ring system with a figure-of-eight structure, and an apparently accessible a position ${ }^{29}$. Here we show that, when FHP is treated with $p$-chloranil in chloroform, an $\alpha-\alpha$ double-bond linked neohexaphyrin dimer $\mathbf{D}$ is obtained in high yield (65\%) (Fig. 2). The directly linked central bipyrrolic subunit in $\mathbf{D}$ is exactly coplanar, and the whole molecule is centrosymmetrical. Also noteworthy is that, relative to FHP, dimer D displays a narrowed highest occupied molecular orbital-lowest unoccupied molecular orbital (HOMO-LUMO) gap, which leads to greater reactivity. Notably, D can react with $\mathrm{MeOH}$ under mild conditions while FHP is inert toward $\mathrm{MeOH}$ under the identical conditions. Upon treating $\mathbf{D}$ with a mixture of tetrahydrofuran (THF)/methanol under either basic or acidic conditions, a pair of isomeric dimers MD1 and MD2 are obtained into which a methoxy moiety is regioselectively attached (Fig. 2). Both MD1 and MD2 are characterized by relatively small HOMO-LUMO energy gaps, red-shifted absorption spectral features, and higher reactivity, compared to the FHP monomer. As a result, all the three dimers can be readily decomposed to give monomeric daughter products under mild conditions. Importantly, the initial insertion reaction that serves to convert $\mathbf{D}$ to $\mathbf{M D 1}$ and $\mathbf{M D 2}$ is relatively rapid and apparently selective for $\mathrm{MeOH}$ over other common alcohols, including ethanol. It also gives rise to a dramatic color change, from green to brown, that allows methanol to be differentiated from other alcohols visually without need for elaborate instrumentation.

Methanol is a key industrial chemical and a recognized human toxin. It is used as a denaturant in laboratory and industrial-grade ethanol but can also be present in distilled beverages whose quality is not carefully controlled ${ }^{30,31}$. Not surprisingly, therefore, considerable effort has been devoted to the problem of sensing methanol in the presence of ethanol and other potentially competing analytes ${ }^{32,33}$. Currently, physical techniques, such as gas chromatography ${ }^{34}$, Fourier transform infrared spectroscopy ${ }^{35}$, and Raman spectroscopy ${ }^{36}$, define the gold standard for methanol sensing. However, these methods rely on relatively expensive instrumentation. Electrochemical and bioanalytical approaches based on nanomaterials are also known 37,38 . In addition, several chemical indicators have been developed in recent years 39,40 . However, the number of bona fide colorimetric sensors and reaction-based indicators for methanol that allow its presence to be detected through simple color changes remain limited and 
a
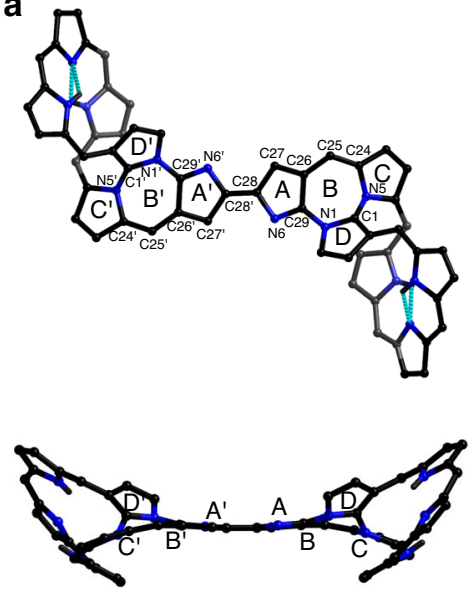

b
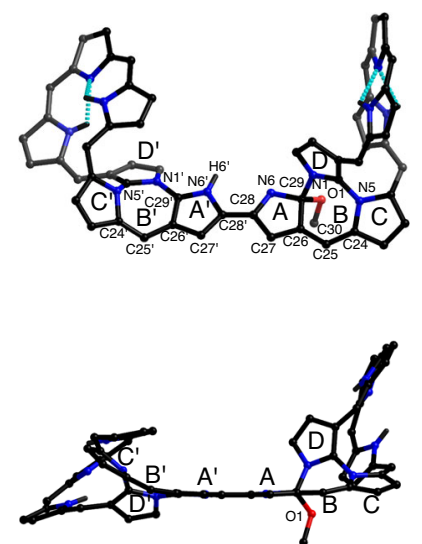

C
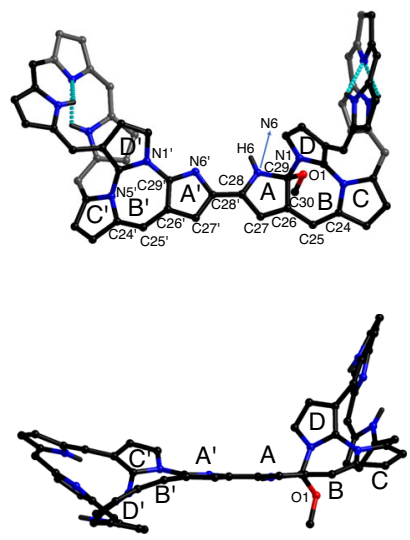

d
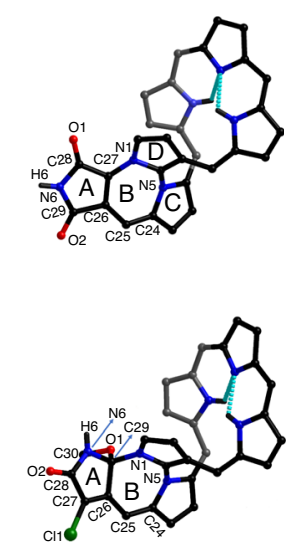

Fig. 3 Crystal structures of the dimers and monomers considered in this study. Complementary views of the molecular structures of D (a), MD1 (b), and MD2 (c) and molecular structures of $\mathbf{F H P O}_{\mathbf{2}}$ (d, top) and FHPMOCI (d, bottom). Pentafluorophenyl substituents, solvents, and the hydrogen atoms attached to the carbon atoms are omitted for clarity. The dotted lines represent intramolecular hydrogen bonds inferred on the basis of the metric parameters. $\mathrm{N}, \mathrm{O}$, and $\mathrm{Cl}$ atoms are denoted with balls of blue, red, and green colors, respectively. $\mathrm{C}$ and $\mathrm{H}$ atoms (linked to $\mathrm{N}$ atoms) are denoted with black balls, with smaller balls used for the latter.

selectivity remains an issue $e^{41-43}$. It is thus possible that the "Twisted-Planar-Twisted" dimer D may have a role to play in addressing this need.

Reaction-based chemosensors are usually based on analytespecific reactions involving appropriately chosen functional groups $^{44,45}$. The post-synthetic functionalization at a specific position of a macrocycles, such as a porphyrinoid, could provide an alternative approach. However, such transformations are usually challenging to implement due to the low selectivity or/and poor yields ${ }^{46}$. As a matter of fact, synthetic macrocycles used as analyte-specific sensors are usually based on supramolecular interactions like weak coordination bonds, hydrogen bonding, and electrostatics ${ }^{44}$, rather than reactivity. In contrast, the rational design and construction of macrocycles incorporating highly reactive $\mathrm{N}$-confused pyrrole units with biased reactivity that can serve as reaction-based chemosensors represents an all but unexplored area of research. As detailed below, the "Twisted-Planar-Twisted" system D may serve as a model for this type of putative reaction-based indicator.

\section{Results}

Synthesis of dimers. When FHP was treated with 5 equivalents of tetrachloro- $p$-benzoquinone ( $p$-chloranil) under reflux in chloroform for $24 \mathrm{~h}$ (Fig. 2), dimer D was obtained as a lightgreen powder in $65 \%$ yield. The high-resolution mass spectrum (HRMS) of D revealed a molecular ion peak at 2561.1683 (Supplementary Fig. 13, see Supporting Information), assignable to a homodimer of FHP produced formally through the elimination of four hydrogen atoms. The ${ }^{1} \mathrm{H}$ spectrum of $\mathbf{D}$ in $\mathrm{CDCl}_{3}$ is characterized by features consistent with a symmetrical structure (Supplementary Fig. 1). Moreover, a singlet at $6.95 \mathrm{ppm}$ corresponding to the $\beta$ protons on the two bridging pyrrole subunits is seen, as would be expected for a dimer linked via the neighboring a-pyrrolic positions. No signal corresponding to the latter protons is seen in $\mathbf{D}$, in contrast to what is observed for FHP. Four $\mathrm{NH}$ proton resonances are seen at $\delta=11.29$ and $9.09 \mathrm{ppm}$, respectively, as expected given the proposed structure.

Upon treating FHP with $p$-chloranil in a mixture of chloroform and methanol $(4 / 1, v / v)$ under reflux and an $\mathrm{N}_{2}$ atmosphere, a heterodimer MD1 and its isomer MD2 were separated as brown solids in 18 and 2\% yield, respectively. The HRMS of MD1 exhibits a molecular ion peak at $m / z=2592.1881$ (Supplementary
Fig. 14). This value is ca. $31 \mathrm{amu}$ higher than the corresponding peak in the HRMS of $\mathbf{D}$, leading us to infer that a methoxy moiety is incorporated into the dimer during the oxidative coupling process. The addition of a single methoxy group was expected to disrupt the symmetry of the overall dimeric molecule. Indeed, ${ }^{1} \mathrm{H}$ and ${ }^{13} \mathrm{C}$ nuclear magnetic resonance (NMR) spectroscopic analyses of $\mathrm{MD1}$ in $\mathrm{CDCl}_{3}$ revealed features consistent with this presumed lack of symmetry. For instance, separate signals for the two distinct monomeric units could be observed (Supplementary Figs. 3-5). In addition, the peaks at 3.41 and $51.5 \mathrm{ppm}$, readily assigned to the methoxy substituents, were seen in the ${ }^{1} \mathrm{H}$ and ${ }^{13} \mathrm{C}$ NMR spectra, respectively (Supplementary Figs. 3, 5). Similar HRMS and NMR spectral features were seen for MD2 (Supplementary Figs. 6 and 15). All three dimers proved soluble in a variety of organic solvents, including $\mathrm{CH}_{2} \mathrm{Cl}_{2}, \mathrm{MeCN}$, THF, toluene, acetone, and dimethylformamide (DMF). This high solubility, which is not recapitulated in most Planar-Planar-Planar dimers, may reflect the twisted nature of the FHP unit and an inability to stack effectively in a layer-bylayer fashion.

Dimers MD1 and MD2 can also be obtained directly from dimer $D$. When a common inorganic base, such as $\mathrm{K}_{2} \mathrm{CO}_{3}$, $\mathrm{Cs}_{2} \mathrm{CO}_{3}$, or $\mathrm{KOH}$, was added into a solution of $\mathbf{D}$ in THF and methanol, a rapid color change from green to brown was observed. After stirring for $5 \mathrm{~min}, \mathrm{MD1}$ and MD2 could be isolated in yields of ca. 56 and $41 \%$, respectively. On the other hand, when common acids, such as $\mathrm{HCl}, \mathrm{H}_{2} \mathrm{SO}_{4}$, TFA, and $p$ $\mathrm{TsOH}$, were used instead of the base, MD1 and MD2 were obtained in yields of ca. 63 and 15\%, respectively. While not studied in detail, the relatively higher yields of MD1 and the increased MD1/MD2 ratios under acidic conditions can be rationalized in terms of slightly different mechanisms that are considered operative under these disparate conditions (Supplementary Figs. 20 and 21).

In contrast to dimer $\mathbf{D}$, the corresponding monomer FHP was not observed to react with $\mathrm{MeOH}$ under similar conditions, even if the reaction time was prolonged from $5 \mathrm{~min}$ to a few days. The higher reactivity of $\mathbf{D}$ is considered to reflect the dramatically narrowed HOMO-LUMO gap and the strong electron-deficient character of the central bipyrrolic unit (vide infra), both of which are expected to facilitate nucleophilic attack by methanol. 
Decomposition of the dimers to monomers. Although FHP is quite stable in $\mathrm{MeOH}$ and other common organic solvents in the presence of air, dimers $\mathrm{D}, \mathbf{M D 1}$, and MD2 are much more reactive and tend to decompose to afford monomers. However, the symmetrical dimer $\mathbf{D}$ and the unsymmetrical dimers MD1/ MD2 exhibit quite different decomposition reactivities. In particular, $\mathrm{Ag}(\mathrm{I})$ salts $\left(\mathrm{CF}_{3} \mathrm{COOAg}, \mathrm{AgPF}_{6}, \mathrm{AgNO}_{3}\right.$, etc. $)$, anhydrous $\mathrm{FeCl}_{3}, \mathrm{DDQ}$, and even air $\left(\mathrm{O}_{2}\right)$ can induce the decomposition of D to give monomer $\mathbf{F H P O}_{2}$ (Fig. 2), along with other unidentified side products. The reaction is not clean and the best yield of $\mathbf{F H P O}$, obtained when $\mathrm{CF}_{3} \mathrm{COOAg}$ was used as the oxidant, was no higher than $8 \%$. The ${ }^{1} \mathrm{H}$ NMR spectrum of FHPO $_{2}$ revealed the absence of a methoxy moiety within the expected 3.0-4.0 ppm chemical shift range (Supplementary Fig. 7). HRMS analysis $\left(m / z=1313.0767,[\mathrm{M}+\mathrm{H}]^{+}\right.$; Supplementary Fig. 16) provided support for the conclusion that two oxygen atoms are incorporated into $\mathbf{D}$ to give $\mathbf{F H P O}_{2}$ during the oxidative cleavage process. In contrast to what is seen for $\mathbf{D}$, under similar reaction conditions both MD1 and MD2 undergo decomposition to afford a methoxy-bearing neo-fused hexaphyrinone FHPMO in relatively clean fashion. For instance, when MD1 and MD2 were treated separately with 8 equivalents of $\mathrm{CF}_{3} \mathrm{COOAg}$ in $\mathrm{CH}_{2} \mathrm{Cl}_{2} / \mathrm{MeOH}$, FHPMO was obtained in high yield (ca. $92 \%$ in both cases). Electrospray ionization-HRMS analysis $\left(m / z=1329.1089,[\mathrm{M}+\mathrm{H}]^{+}\right.$; Supplementary Fig. 17) revealed that FHPMO incorporates an oxygen atom in addition to a methoxy moiety. A strong peak ascribed to the methoxy moiety was observed at $3.47 \mathrm{ppm}$ in the ${ }^{1} \mathrm{H}$ NMR spectrum (Supplementary Fig. 11). We thus conclude that the silver(I)promoted decomposition reactions of the unsymmetrical dimers MD1 and MD2 are much simpler and cleaner than those of the homodimer $\mathbf{D}$, a difference attributed to the effect of the methoxy group. Furthermore, FHPMO was found to undergo a highly regioselective monochlorination reaction to afford FHPMOCl in $78 \%$ yield when treated with anhydrous $\mathrm{FeCl}_{3}$ in chloroform/ methanol. Evidence for the formation of FHPMOCl came from HRMS analyses $\left(m / z=1363.0712,[\mathrm{M}+\mathrm{H}]^{+}\right.$, Supplementary Fig. 18), ${ }^{1} \mathrm{H} /{ }^{13} \mathrm{C}$ NMR spectroscopy (Supplementary Figs. 11 and 12 ), and a single crystal X-ray diffraction analysis (vide infra).

Single crystal structures. Further insight into the structures of D, MD1, MD1, FHPO 2 , and FHPMOCl came from X-ray diffraction analyses. As shown in Fig. 3, D displays a "Z"-shaped trans configuration with the "figure-of-eight" conformation of the hexaphyrin subunits reserved in the dimer (Fig. 3a). Dimer D is centrosymmetric and the two units are bridged by the C28-C28' double bond (1.393(8) Å) (Supplementary Fig. 29; for numbering and subunit labels, see Fig. 3). The two monomeric units are linked through the coplanar bipyrrolic bridging unit A-A', with pyrroles $\mathrm{C}\left(\mathrm{C}^{\prime}\right)$ and $\mathrm{D}\left(\mathrm{D}^{\prime}\right)$ slightly tilted off this plane as reflected in the relatively small dihedral angles of $4.5^{\circ}$ and $14.4^{\circ}$, respectively. The relative planarity of the multiply fused constituent cycles (C-D-B-A-A'-B'-D'-C') was expected to favor effective $\pi$ conjugation and associated electronic coupling between the two hexaphyrin subunits. To the extent this proved true, it would be expected to have a significant effect on the electronic and electrochemical features, as in fact is seen by experiment (vide infra).

In contrast to $\mathbf{D}$, the unsymmetrical heterodimers MD1 and MD2 adopt cis configurations about the bridging C28-C28', for which bond lengths of 1.416(5) and 1.379(6) $\AA$ are observed, respectively. The difference in the latter values is ascribed to the presence of what may be considered as being formal single and double bonds, respectively (Fig. 3b, c and Supplementary Figs. 30 and 31). The bipyrrolic units A-A' in MD1 and MD2 both show good planarity and are characterized by small dihedral angles of $4.6^{\circ}$ and $5.9^{\circ}$, respectively.

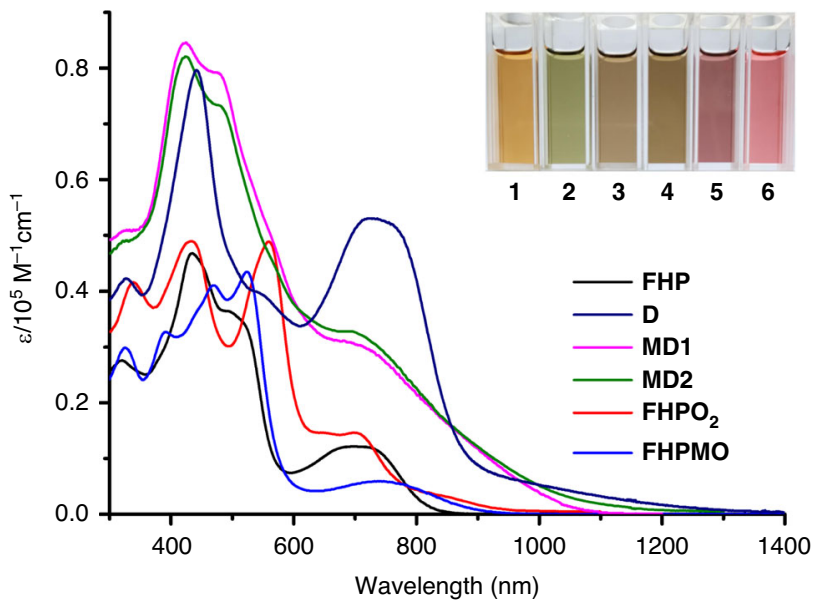

Fig. $4 \mathrm{UV} / \mathrm{vis} / \mathrm{NIR}$ absorption spectra of the dimers and monomers considered in this study as recorded in $\mathbf{C H}_{\mathbf{2}} \mathbf{C l}_{\mathbf{2}}$. The inset shows photographs of the corresponding solutions in $\mathrm{CH}_{2} \mathrm{Cl}_{2}\left(1.0 \times 10^{-5} \mathrm{M}\right.$ for all compounds). Here the numbers 1-6 refer to FHP, D, MD1, MD2, FHPO, and FHPMO, respectively.

In both unsymmetrical dimers, a methoxy group is attached to one of the hexaphyrin units. In MD1, this group is attached at $\mathrm{C} 29$, which is best described as being an $\mathrm{sp}^{3}$-hybridized carbon center. The surrounding $\mathrm{C}-\mathrm{N}, \mathrm{C}-\mathrm{O}$ and $\mathrm{C}-\mathrm{C}$ bond lengths are in the range of $1.40-1.54 \AA$, typical for single bonds. It is noteworthy that pyrrole $\mathrm{D}$ is tilted from the A-A' plane with a relatively large dihedral angle of $80.6^{\circ}$. On the other hand, the methoxy-free subunit of MD1 is more planar, with pyrroles C' and D' tilted from A-A' at smaller dihedral angles of $37.9^{\circ}$ and $36.8^{\circ}$, respectively. In $\mathbf{M D 2}$, the corresponding values are $31.9^{\circ}$ and $34.8^{\circ}$, respectively, slightly smaller than those observed for MD1. These dihedral angles are all larger than the corresponding values in $\mathbf{D}$, indicative of greater distortion.

Ultraviolet/visible/near-infrared (UV/vis/NIR) absorption spectra. Compared with FHP, the absorption bands of the dimers tail further into the red (Fig. 4). They are also roughly twice as intense, as might be expected for dimers incorporating twice the number of chromophores on a per mole basis. In the case of $\mathbf{D}$, a Soret-like band with a sharp peak centered at ca. $442 \mathrm{~nm}$ is seen, along with a broad and very intense Q-like band centered at $727 \mathrm{~nm}$ that tails out to ca. $1300 \mathrm{~nm}$. In contrast, the spectrum of the unsymmetrical dimer MD1 is characterized by a split Soretlike band with local maxima centered at 423 and $479 \mathrm{~nm}$, as well as a shoulder at ca. $693 \mathrm{~nm}$. The absorption edge of MD1 extends to $\mathrm{ca} .1070 \mathrm{~nm}$, a reduction in the tailing compared to $\mathbf{D}$ that may reflect interruption in the local conjugation as a consequence of the incorporated methoxy group. The absorption spectrum of heterodimer MD2 resembles that of MD1. However, the absorption edge of MD2 approaches ca. $1250 \mathrm{~nm}$, an observation consistent with the more extended conjugation expected for MD2 as the result of the double bond link that connects the two monomeric units (Supplementary Fig. 34). The absorption bands of $\mathrm{FHPO}_{2}$ and FHPMO roughly mirror those of FHP, consistent with their monomeric structures. However, their absorption features tail a bit further to the red (to ca. 960 and $910 \mathrm{~nm}$ for FHPO $_{2}$ and FHPMO, respectively). In addition, the Soret-like feature is strongly split in the case of $\mathbf{F H P O}_{2}$.

Electrochemistry. The electrochemical properties of the dimers and monomers were studied using cyclic voltammetry $(\mathrm{CV})$ and differential pulse voltammetry (DPV) in $\mathrm{CH}_{2} \mathrm{Cl}_{2}$ containing $0.1 \mathrm{M}$ 
a

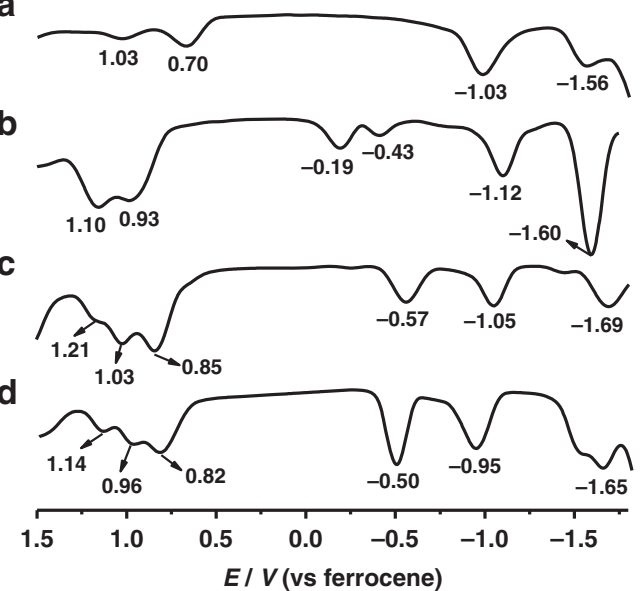

Fig. 5 Electrochemical studies of the dimers considered in this study and the monomer FHP. Differential pulse voltammograms (DPV) of FHP (a), D (b), MD1 (c), and MD2 (d). DPV measurements were conducted in anhydrous $\mathrm{CH}_{2} \mathrm{Cl}_{2}$ containing $0.1 \mathrm{M}$ tetra-n-butylammonium perchlorate $\left(\mathrm{TBAClO}_{4}\right)$ as the supporting electrolyte. A three-electrode cell was used: a glassy carbon (working electrode), platinum wire (counter electrode), and $\mathrm{Ag} / \mathrm{AgCl}$ (reference electrode). Ferrocene/ferrocenium ( $\mathrm{Fc} / \mathrm{Fc}-$ ) was used as an external reference.

tetra- $n$-butylammonium perchlorate $\left(\mathrm{TBAClO}_{4}\right)$ as the supporting electrolyte (Fig. 5 and Supplementary Figs. 27 and 28). All the three dimers, D, MD1, and MD2, displayed electrochemical features that were noticeably different from those of FHP. Homodimer $\mathbf{D}$ displays two partially overlapped quasi-reversible oxidation waves at 0.93 and $1.10 \mathrm{~V}$, which are anodically shifted compared with FHP $(0.70,1.03 \mathrm{~V})$. Four different reduction processes were observed and the potentials for the first two are anodically shifted to -0.19 and $-0.43 \mathrm{~V}$, respectively, compared to the corresponding value of $-1.03 \mathrm{~V}$ observed for FHP. The positively shifted reductions are considered to reflect the more electron-deficient nature of $\mathbf{D}$. The first and second reduction potentials can be viewed as a split reduction couple, and the large potential difference between them $\left(\Delta E_{\text {red }}=0.24 \mathrm{~V}\right)$ is taken as evidence of effective electronic communication ${ }^{47,48}$ between the two hexaphyrin subunits mediated by the planar bipyrrolic linking unit. As true in the case of the UV-vis-NIR spectral studies (vide supra), the unsymmetrical dimers MD1 and MD2 demonstrate similar electrochemical features. Three slightly anodically shifted oxidation waves (versus two processes for FHP and D) were observed for these unsymmetrical dimers, with the values for first oxidation potentials being 0.85 and $0.82 \mathrm{~V}$, respectively. Similar to what proved true for $\mathbf{D}$, the first reduction waves of MD1 and MD2 $(-0.57,-0.50 \mathrm{~V})$ are anodically shifted compared to FHP $(-1.03 \mathrm{~V})$, although to a lesser extent than D $(-0.19 \mathrm{~V})$. Based on the DPV data, the electrochemical HOMO-LUMO gaps between the first oxidation and first reduction potentials of $\mathrm{D}, \mathrm{MD1}$, and MD2 were estimated to be $1.12,1.42$, and $1.32 \mathrm{~V}$, respectively (Supplementary Table 1 ). This observation is consistent with what was seen for the tailing absorption band edges. The inferred HOMO-LUMO gaps for the dimers are much smaller than that for FHP $(1.73 \mathrm{eV})$. Again, this is consistent with effective $\pi$-conjugation occurring to a greater or lesser extent throughout the three dimers.

Theoretical calculations. Density functional theory (DFT) calculations using the Gaussian 16 program package $^{49}$ were performed on the dimers in an effort to understand further their distinct structures and properties. First, the energies of both the putative cis and trans isomers were calculated at different energy levels. The B3LYP/6-31G(d) ${ }^{50}$ and extended basis sets $(6-31 G(d, p)$ and $6-311 \mathrm{G}(\mathrm{d}, \mathrm{p}))^{51,52}$ all gave similar values for the energy barriers between isomers D/D-a and MD1/MD1-a/MD2/MD2-a, respectively. The use of the $\mathrm{BMK}$ functional ${ }^{53}$ gave similar results as the B3LYP functional. In addition, the Grimme D3 correction $^{54}$ was included for the B3LYP functional; this gave the same trend (Supplementary Tables 5 and 6). It was found that the relative energy of the trans dimer (D) is $17.3 \mathrm{~kJ} / \mathrm{mol}$ lower than the corresponding cis isomer D-a (Fig. 6a). Such a computational finding is consistent with the observation that $\mathbf{D}$ is obtained as the sole product and in relatively high yield when FHP is subject to oxidative coupling. In contrast, the isomeric cis dimers (MD1 and MD2) were both calculated to lie at much lower energies than their corresponding trans dimers MD1-a and MD2-a; again, this finding agrees well with experiment, specifically the finding that neither MD1-a nor MD2-a could be detected under the conditions of the methanol insertion reaction (Fig. 6b). On the other hand, the energy of MD2 is slightly higher than its isomer MD1 (by $+2.1 \mathrm{~kJ} /$ $\mathrm{mol}$ ), roughly consistent with the observation that the synthetic yields for MD1 are higher than MD2 under the various synthetic conditions tested in the context of this study (vide supra).

Notably, the frontier molecular orbitals of D, MD1, and MD2 are all remarkably different (Fig. $7 \mathrm{a}-\mathrm{c}$ ). For $\mathbf{D}$, the LUMO orbital is mainly distributed over the central multiply fused unit showing a relatively low energy of $-3.78 \mathrm{eV}$. This results in a narrow HOMO-LUMO gap of $1.26 \mathrm{eV}$, which is ca. $0.74 \mathrm{eV}$ smaller than that of FHP. This is taken as evidence of the pronounced electron-deficient character of the two bridging imino-type pyrrolic units (A-A'), an inference that, in turn, explains the susceptibility to nucleophilic attack by $\mathrm{MeOH}$ (or its alkoxide anion) to form MD1/2. Such an inference is consistent with the positive shifts in the first two reduction waves seen in $\mathbf{D}$ (vide supra). The HOMO and HOMO-1 of $\mathbf{D}$ are almost degenerate (orbital energies: -5.04 and $-5.06 \mathrm{eV}$, respectively) with the orbitals extended over the two hexaphyrin macrocycles except for the central multiply fused units. The high symmetry and relatively strong interaction between the two hexaphyrin units may be responsible for the near-degeneracy ${ }^{54-57}$. For MD1, the HOMO and LUMO are both delocalized over the methoxycontaining hexaphyrin unit. Specifically, the LUMO is delocalized over the whole hexaphyrin macrocycle, while the HOMO is distributed over the macrocycle except for the fused tetracyclic unit (ABCD rings; see Fig. 3b). No evidence of degenerate orbitals is seen for the HOMO of MD1, which may reflect the reduced symmetry of this unsymmetrical dimer relative to $\mathbf{D}$. In the case of MD2, the LUMO orbital is mainly located on the methoxycontaining hexaphyrin moiety, as well as the pyrrolic unit ( $\left.A^{\prime}\right)$ of the other hexaphyrin moiety. The HOMO, however, is mainly distributed over the methoxy-free hexaphyrin unit. The obviously different orbital distributions of MD1 and MD2 can be rationalized by the fact that the conjugation pathway in MD1 is disturbed, which results in relatively weak interaction between the two hexaphyrin units, while a fuller conjugated pathway exists in MD2 (Supplementary Fig. 34).

The energy levels of the LUMO orbitals for MD1 and MD2 are dramatically negatively shifted to -3.09 and $-3.27 \mathrm{eV}$, respectively, relative to FHP $(-2.90 \mathrm{eV})$. The corresponding HOMO levels are located at -4.84 and $-4.75 \mathrm{eV}$, respectively. This represents a slight positive shift compared to FHP $(-4.90 \mathrm{eV})$. As a result of the dramatically lowered LUMO orbitals, the HOMO-LUMO gaps of D, MD1, and MD2 are 1.26, 1.75, and $1.48 \mathrm{eV}$, respectively (Fig. 6c). They are thus much smaller than those of FHP $(2.00 \mathrm{eV})$. These reductions in the HOMO-LUMO gaps are fully consistent with the trends inferred from the spectroscopic and electrochemical analyses discussed above. 
a

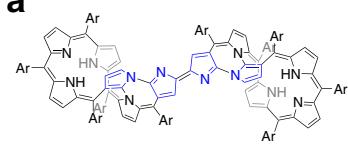

D

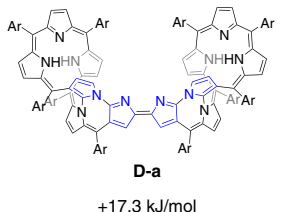

$+17.3 \mathrm{~kJ} / \mathrm{mol}$ b
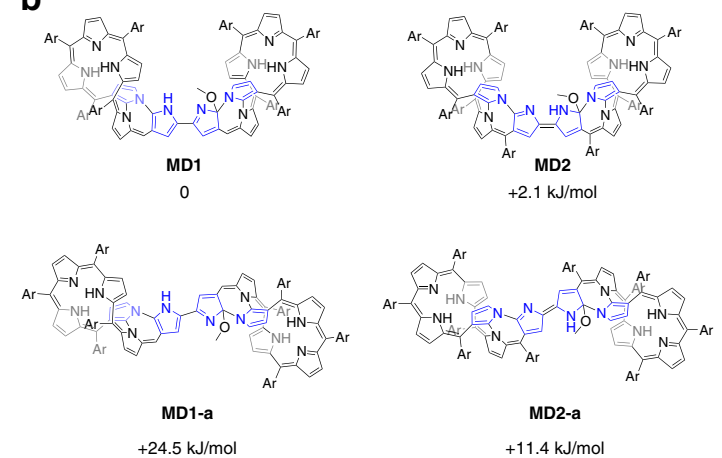

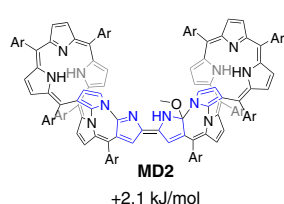

$+11.4 \mathrm{~kJ} / \mathrm{mo}$ c

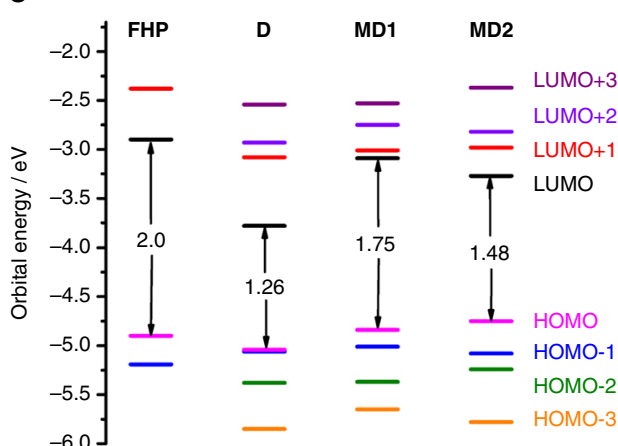

Fig. 6 DFT-calculated relative/orbital energies at the B3LYP/6-31G(d) level of theory. a Computationally derived relative energies of D-a; and the putative cis isomer of D; b computational relative energies of the unsymmetrical dimers MD1, MD2, and the possible trans isomers MD1-a and MD2-a; c selected molecular orbital energies for FHP and dimers D, MD1, and MD2. Ar $=\mathrm{C}_{6} \mathrm{~F}_{5}$. The blue-colored pyrrolic units in a and $\mathbf{b}$ represent confused pyrroles that are linked with adjacent pyrroles or meso-carbons through $\mathrm{C}_{\alpha}-\mathrm{C}_{\beta}$ or $\mathrm{N}-\mathrm{C}_{\beta}$ atoms.

As mentioned above, the experimental absorption spectra of the three dimers appear to be very simple in the low energy region beyond $600 \mathrm{~nm}$. These features are quite different from those of traditional porphyrin dimers ${ }^{55-57}$. To provide further insight into these observations and understand the dramatically different absorption features for the three dimers, time-dependent (TD) DFT calculations were carried out. At first, the energy of the $\mathrm{S}_{1}$ state $(\mathrm{nm})$ for the dimers were calculated by different functionals (B3LYP, PBE0, $\omega$-B97XD, M062X, CAM-B3LYP, $\mathrm{BMK}$, etc.) using the $6-31 \mathrm{G}(\mathrm{d})$ basis set and the polarizable continuum model (PCM) model (dichloromethane (DCM) as a solvent) (Supplementary Table 7). Relative to the observed absorption spectra, both the B3LYP 58 and $\mathrm{PBE}^{59}$ functionals underestimate the energy of the first $S_{0} \rightarrow S_{1}$ transition for $\mathbf{D}$ and MD2. On the other hand, the long-range corrected CAM-B3LYP ${ }^{60}$ functional slightly overestimates the energy of the $\mathrm{S}_{0} \rightarrow \mathrm{S}_{1}$ electronic transition for MD1, while it overestimates the energy of the $S_{0} \rightarrow S_{1}$ electronic transition for dimer $\mathbf{D}$. For the $\omega-\mathrm{B}^{2} 7 \mathrm{XD}^{61}$ and M062X ${ }^{62}$ functionals, the difference between the $S_{0} \rightarrow S_{1}$ electronic transition of MD1 and MD2 is not fully revealed. In contrast, the BMK functional, which usually gives improved accuracy for transition state barriers ${ }^{53}$, gave relatively reasonable results for all the three dimers compared to the other functionals employed. For $\mathbf{D}$, the exceptionally strong and broad absorption band in the $600-900 \mathrm{~nm}$ region can be rationalized in terms of a HOMO-2 $\rightarrow$ LUMO transition with quite a large oscillator strength $(f=1.81)$. Both the HOMO-2 and LUMO orbitals are localized on the bridging bipyrrolylidene fragment (Fig. 7a), indicating that this band mainly originates from a localized $\pi-\pi^{*}$ transition, instead of an intramolecular charge transfer (ICT) from the hexaphyrin core to the central multiply fused linking unit ${ }^{63-65}$. The Q-like band of compound $\mathbf{D}$ corresponds to the quasidegenerated $S_{0} \rightarrow S_{1}$ and $S_{0} \rightarrow S_{2}$ transitions, and the oscillator strengths $(f=0.26 / 0.005)$ are much smaller than those of the HOMO- $\rightarrow$ LUMO transition $(f=1.81)$ (Supplementary Fig. 35 and Supplementary Table 9). Hence, the lower energy band is observed to be submerged within the strong broad band in the $600-900 \mathrm{~nm}$ region. As a result, no clearly resolved Q-like bands could be observed in contrast to other reported dimers characterized by different linking modes ${ }^{55}$.

As can be seen from Fig. 7b, the occupied and unoccupied molecular orbitals of MD1 are mainly localized on either one of the hexaphyrin units. Therefore, the calculated lowest energy transitions of MD1 at 817 and $698 \mathrm{~nm}$, mainly corresponding to $\mathrm{HOMO}-1 \rightarrow \mathrm{LUMO}$ and $\mathrm{HOMO} \rightarrow \mathrm{LUMO}+1$ excitations, respectively, are both characteristic of weak oscillator strengths $(f \approx 0.1)$ because of the ICT nature associated with the electronic distribution (Supplementary Fig. 36 and Supplementary Table 10). On the other hand, the high energy transitions in the range of $400-600 \mathrm{~nm}$ (Soret-like band) demonstrate relatively larger oscillator strengths $(f=0.3-0.7)$. Therefore, absorptions from the low energy transitions are submerged under the higher energy ones. As a result, MD1 exhibits only a shoulder peak at ca. $693 \mathrm{~nm}$ instead of well-resolved Q-like bands as observed for the traditional porphyrin dimers ${ }^{55-57}$. Similar to MD1, the lowest energy excitations of MD2 also correspond to HOMO- $1 \rightarrow$ LUMO and $\mathrm{HOMO} \rightarrow \mathrm{LUMO}+1$ transitions (Supplementary Fig. 37 and Supplementary Table 11). Notably, the HOMO-1 orbitals are located over both of the hexaphyrin fragments, and the HOMO- $\rightarrow$ LUMO transition is symmetry allowed, leading to a larger oscillator strength (0.37) than that of MD1, a finding that serves to rationalize the slightly stronger Q-like band and longer wavelength band edge for MD2 in comparison with MD1.

Methanol response. As mentioned above, a dramatic solution color change from light green to brown was observed accompanying with the conversion from $\mathrm{D}$ to $\mathrm{MD1} / \mathbf{2}$. As a matter of fact, solutions of $\mathbf{D}$ in THF under basic conditions are very sensitive to methanol, while the response to ethanol and other alcohols is considerably slower. Presumably, this reflects the somewhat lowered nucleophilicity and greater steric hindrance of these higher alcohols. In principle, this reactivity difference could be used to differentiate methanol from other alcohols. As a test of this hypothesis, various alcohols $(3.0 \mu \mathrm{L}$ corresponding to ca. $25 \mathrm{mM}$ after dilution) were added, respectively, into THF solutions of $\mathbf{D}\left(1.3 \times 10^{-2} \mathrm{mM}\right.$ in $\left.3.0 \mathrm{~mL}\right)$ in the presence of excessive $\mathrm{NaOH}_{(\mathrm{s})}(10 \mathrm{mg})$. Under these conditions, a discernible color change was found for the methanol sample within $5 \mathrm{~min}$ (Fig. 8). However, no obvious color change was observed in the case of any of the other alcohols during the same 5-min time period. Kinetic studies, involving the response of $\mathbf{D}$ toward methanol and ethanol as a function of time, were carried and provide support for the fact that these two alcohols give rise to remarkably different reaction rates (Supplementary Figs. 22 and 23). Notably, when the amount of added methanol was decreased to $0.50 \mu \mathrm{L}$ (corresponding to ca. $4.2 \mathrm{mM}$ after dilution), a color change from green to light brown still occurs, although $24 \mathrm{~h}$ were required to effect essentially complete conversion (Supplementary Figs. 24 and 25). On this basis, we conclude that methanol may be readily differentiated from other alcohols by simply monitoring whether the distinctive color change is produced during a set time period. Furthermore, the detection limit ${ }^{66}$ of methanol in THF was 
a

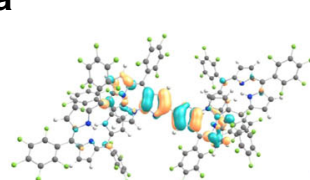

LUMO $(-3.78 \mathrm{eV})$

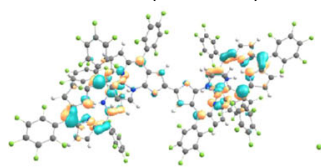

$\mathrm{HOMO}(-5.04 \mathrm{eV})$

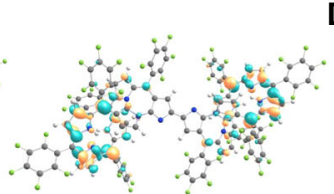

$\mathrm{LUMO}+1(-3.08 \mathrm{eV})$

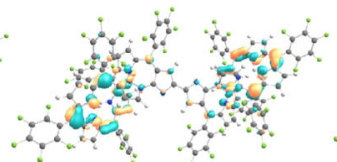

HOMO-1 (-5.06 eV)
D

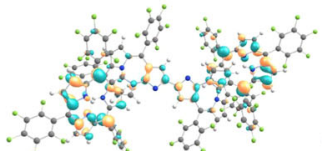

LUMO+2 (-2.93 eV)

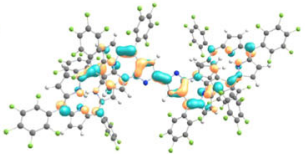

HOMO-2 (-5.38 eV)

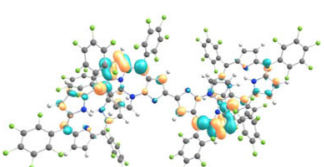

LUMO+3 $(-2.54 \mathrm{eV})$

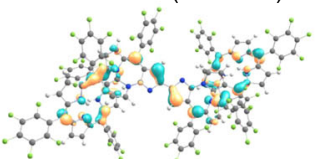

HOMO-3 (-5.85 eV) b

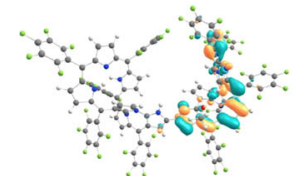

LUMO (-3.09 eV)

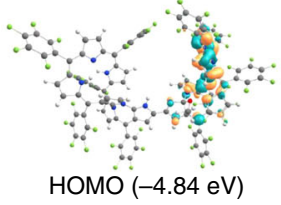

c

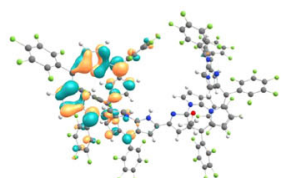

LUMO+1 $(-3.01 \mathrm{eV})$

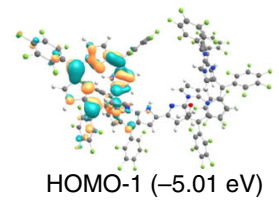

MD1

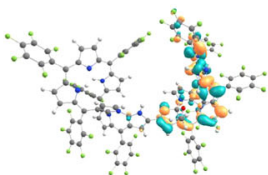

LUMO+2 (-2.75 eV)
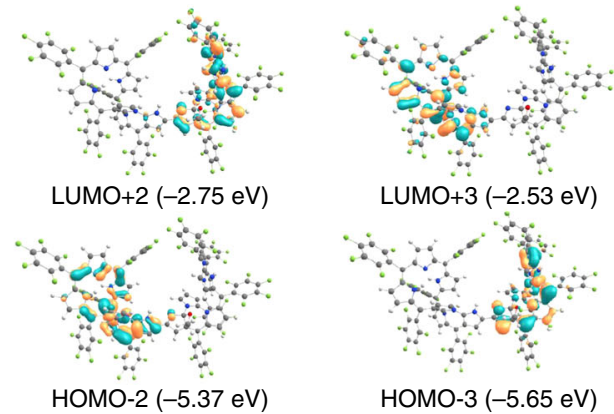

LUMO+3 (-2.53 eV)

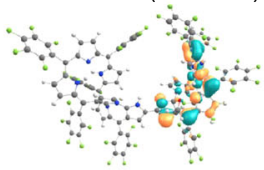

HOMO-3 (-5.65 eV)

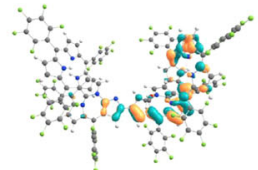

LUMO (-3.27 eV)

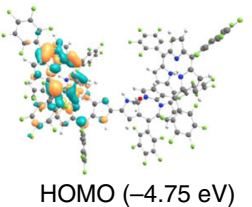

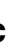

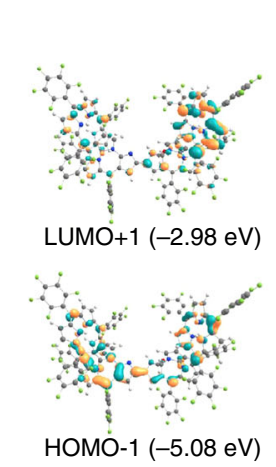

MD2

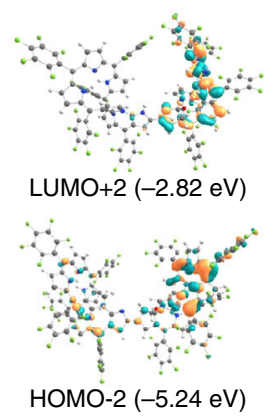

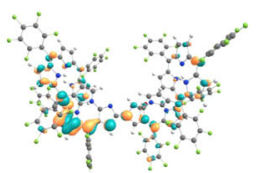

LUMO+3 (-2.37 eV)

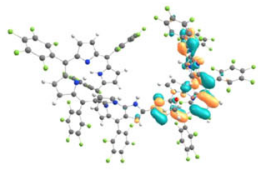

HOMO-3 (-5.78 eV)

Fig. 7 Selected contour plots of the DFT-optimized structures and various orbitals for the compounds of this study. a-c stands for D, MD1, and MD2, respectively. The corresponding energy values are included in parentheses. The isosurface control value is 0.03 a.u. The occupied molecular orbitals are highlighted with blue and orange colors. $\mathrm{C}, \mathrm{N}, \mathrm{O}, \mathrm{H}$, and $\mathrm{F}$ atoms are denoted with balls of gray, red, white, and green colors, respectively.

a
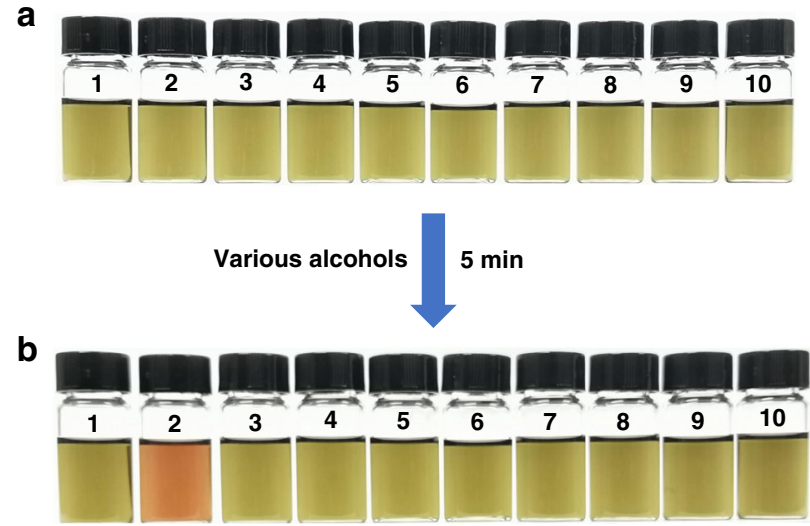

Fig. 8 Photographs of the $D$ solutions in response to various alcohols. a Vials containing $\mathbf{D}\left(1.3 \times 10^{-5} \mathrm{~mol} / \mathrm{L}\right)$ and $\mathrm{NaOH}_{(\mathrm{s})}(10 \mathrm{mg})$ in $\mathrm{THF}$ $(3.0 \mathrm{~mL}) . \mathbf{b}$ The same vials as in $\mathbf{a}$ after adding $3.0 \mu \mathrm{L}$ of the following alcohols (from left to right and numbered 1-10): none, methanol, ethanol, 1-propanol, 2-propanol, 1-butanol, 2-butanol, phenylcarbinol, ethylene glycol, and glycerol, respectively. estimated to be ca. $0.037 \mathrm{mM}$ based on the 24 -h reaction timeframe (Supplementary Fig. 26). On this basis, we proposed that D may emerge as a promising methanol indicator that can operate in the absence of relatively expensive analytical instrumentation.

\section{Discussion}

Oxidative coupling of an unusual twisted neo-fused hexaphyrin (FHP) with a protruding $\mathrm{N}$-confused pyrrole gives rise to the "Twisted-Planar-Twisted" homodimer D with a trans configuration in high yield, leading us to suggest that introducing a reactive subunit into a twisted porphyrinoid is a promising approach for constructing functional dimers or even larger oligomers. Upon adding methanol to the reaction mixture under basic or acid conditions, regioselective addition of a methoxy moiety to $\mathbf{D}$ occurs to afford two isomeric heterodimers, MD1 and MD2, both of which contain cis-type central linkages. A strong interaction between the two hexaphyrin subunits of $\mathbf{D}$ is inferred from its spectral and physical properties and is ascribed to the presence of a coplanar central bipyrrolic linking unit and the roughly coplanar arrangement of the appended neo-fused 
hexaphyrin subunits. Compared with $\mathbf{D}$, reduced intramolecular electronic interactions are seen in the case of MD1 and MD2. These latter isomeric species differ somewhat from one another, reflecting differences in their respective conjugation pathways and molecular conformations. The fact that $\mathbf{D}$ reacts readily and selectively with methanol under readily defined reaction conditions allows for the qualitative differentiation of methanol from ethanol and other alcohols by simple visual means. The associated sensing function could prove useful in detecting methanol in either an industrial or beverage-related setting. Further studies of this possibility are ongoing in our laboratory.

The studies reported here not only illustrate a promising approach to synthesizing porphyrinoid dimers by oxidatively coupling the corresponding monomers through their accessible a-pyrrolic positions but also provide an approach for developing reaction-based chemosensors. The latter potential application takes advantage of the fact that the present dimers allow for biased high reactivity that reflects a synergetic effect of reduced HOMO-LUMO energy gaps and the presence of reactive $\mathrm{N}$ confused units. While further work will be needed to develop the present findings as a potentially generalizable strategy, the approach to sensing detailed here could provide a useful complement to current methods that typically involve the rational design of specific receptors or reactive constructs.

\section{Methods}

General methods. Commercially available solvents and reagents were purchased from Adamas-Beta and used without further purification unless otherwise mentioned. Thin-layer chromatography was carried out on aluminum sheets coated with silica gel $60 \mathrm{~F} 254$ (MERCK). ${ }^{1} \mathrm{H}$ NMR $(400 \mathrm{MHz})$ spectra were obtained using a Bruker AM 400 spectrometer. Chemical shifts are reported relative to tetramethylsilane $(\delta=0)$ in ppm. ${ }^{13} \mathrm{C}$ NMR spectra were recorded at $101 \mathrm{MHz}$ (Bruker AM 400) and the chemical shifts were reported relative to $\mathrm{CDCl}_{3}(\delta=77.00),\left(\mathrm{CD}_{3}\right)_{2} \mathrm{CO}(\delta=29.84)$, or DMSO- $d_{6}(\delta=39.52)$ in ppm; see Supplementary Figs. $1-12$. HRMS analyses were performed using a Waters LCT Premier XE spectrometer or Bruker Solarix XR FTMS instrument for $\mathrm{CH}_{2} \mathrm{Cl}_{2}$ solutions; see Supplementary Figs. 13-18. UV-vis-NIR absorption spectral studies were carried out using a Shimadzu UV2600 spectrophotometer with all spectra being recorded at room temperature.

Syntheses of compounds. Homodimer D was synthesized through a one-pot oxidation of the monomer FHP in methanol-free solvents, in yields of ca. $65 \%$. The unsymmetrical dimers MD1 and MD2 can be obtained either from FHP or D, both in the presence of methanol. The monomers $\mathbf{F H P O}_{2}$ and FHPMO were obtained by the direct oxidation of D and MD1 (MD2), respectively (see Supplementary Methods). The target compounds were fully characterized by single crystal X-ray diffraction analyses, as well as by NMR spectroscopy and HRMS analyses (Supplementary Methods).

Crystallography. Single crystals of D were obtained by the slow recrystallization from the DCM/heptane solution; crystals of D1 and FHPMOCl were obtained by the slow recrystallization from their DCM/octane solutions, and crystals of D2 and FHPMO $\mathrm{M}_{2}$ were obtained by the slow evaporation of their acetonitrile/water solutions. Single crystal X-ray analyses were performed on a SMART APEX equipped with CCD detector (Bruker) using MoKa (graphite, monochromated, $\lambda=0.71073 \AA$ ) radiation or CuKa (graphite, monochromated, $\lambda=1.54178 \AA$ ) radiation. The structures were solved by the direct method of SHELXS-97/2014/ 2018 and refined using the SHELXL-97/2014 or Olex2 1.2 program ${ }^{49,67,68}$. The positional parameters and thermal parameters of non-hydrogen atoms were refined anisotropically on $F^{2}$ by the full-matrix least-squares method. Hydrogen atoms were placed at calculated positions and refined riding on their corresponding carbon atoms. Detailed crystallographic data are provided in Supplementary Tables 2 and 3.

Electrochemical measurements. CV and DPV studies were carried out at $298 \mathrm{~K}$ using a CHI-730C Electrochemical Workstation. A three-electrode cell consisting of a glassy carbon (working electrode), platinum wire (counter electrode), and Ag/ $\mathrm{AgCl}$ (reference electrode) attached to a Chi-730D/620E electrochemistry station were used, with the potentials calibrated to the ferrocenium/ferrocene $\left(\mathrm{Fc}^{+} / \mathrm{Fc}\right)$ couple. Absolute DCM $\left(\mathrm{CH}_{2} \mathrm{Cl}_{2}\right)$ was purchased from Adamas-Beta and used as received and $\mathrm{TBAClO}_{4}$ was purchased from Fluka Chemika, recrystallized from ethanol, and dried under vacuum at $40^{\circ} \mathrm{C}$ for at least 1 week prior to use.

Theoretical calculation details. All calculations were carried out using the Gaussian 16 suite of programs ${ }^{49}$. The geometries of all molecules under study were optimized using B3LYP 58 and the $6-31 G(d)$ basis $\operatorname{set}^{50}$ for all the atoms directly. No adjustment to the effective core potential was applied. All the optimized geometries were checked as the real minima on the potential energy surface by means of frequency calculations. At the optimized geometries, TD DFT calculations with the hybrid $\mathrm{BMK}$ functional ${ }^{53}$ were carried out to simulate the absorption spectra. Solvent effects arising from $\mathrm{CH}_{2} \mathrm{Cl}_{2}$ were accounted for using the $\mathrm{PCM}^{69}$.

\section{Data availability}

CCDC 1886415-1886417, 1985682, and 1985685 contain the supplementary crystallographic data for this paper. These data can be obtained free of charge from The Cambridge Crystallographic Data Centre via www.ccdc.cam.ac.uk/data_request/cif. All other data supporting the findings of this study are available from the corresponding authors upon request.

Received: 27 May 2020; Accepted: 24 September 2020; Published online: 20 October 2020

\section{References}

1. Kadish, K. M., Smith, K. M. \& Guilard, R. The Porphyrin Handbook (Academic Press, 2000).

2. Ding, Y., Zhu, W.-H. \& Xie, Y. Development of ion chemosensors based on porphyrin analogues. Chem. Rev. 117, 2203-2256 (2017).

3. Szyszko, B., Białek, M. J., Pacholska-Dudziak, E. \& Latos-Grażyński, L. Flexible porphyrinoids. Chem. Rev. 117, 2839-2909 (2017).

4. Sarma, T. \& Panda, P. K. Annulated isomeric, expanded, and contracted porphyrins. Chem. Rev. 117, 2785-2838 (2017).

5. University of Sheffield \& Chemical Society (Great Britain). Aromaticity: An International Symposium Held at Sheffield on 6th-8th July 1966. Special Publication No. 21 (The Chemical Society London, London, 1966). First reported by Woodward, R. B.

6. $\mathrm{Li}, \mathrm{Q}$. et al. Skeletal rearrangement of twisted thia-norhexaphyrin: multiply annulated polypyrrolic aromatic macrocycles. Angew. Chem. Int. Ed. $\mathbf{5 8}$ 5925-5929 (2019).

7. Tanaka, T. \& Osuka, A. Chemistry of meso-aryl-substituted expanded porphyrins: aromaticity and molecular twist. Chem. Rev. 117, 2584-2640 (2017)

8. Roznyatovskiy, V. V., Lee, C.-H. \& Sessler, J. L. $\pi$-Extended isomeric and expanded porphyrins. Chem. Soc. Rev. 42, 1921-1933 (2013).

9. Burrell, A. K., Cyr, M. J., Lynch, V. \& Sessler, J. L. Nucleophilic attack at the meso position of a uranyl sapphyrin complex. J. Chem. Soc. Chem. Commun. 1710-1713 (1991).

10. Ishizuka, T., Osuka, A. \& Furuta, H. Inverted N-confused porphyrin dimer. Angew. Chem. Int. Ed. 43, 5077-5081 (2004).

11. Brewster, J. T. et al. $\mathrm{UO}_{2}{ }^{2+}$-mediated ring contraction of pyrihexaphyrin: synthesis of a contracted expanded porphyrin-uranyl complex. Chem. Sci. 10, 5596-5602 (2019).

12. Li, C. et al. Tripyrrin-armed isosmaragdyrins: synthesis, heterodinuclear coordination, and protonation-triggered helical inversion. Chem. Sci. 11, 2790-2795 (2020)

13. Kong, J. et al. Modulation of the structures and properties of bidipyrrin zinc complexes by introducing terminal $\alpha$-methoxy groups. Dyes Pigm. 137, 430-436 (2017).

14. Maeda, H., Osuka, A. \& Furuta, H. Trans doubly N-confused porphyrins: $\mathrm{Cu}$ (III) complexation and formation of rodlike hydrogen-bonding networks. J. Am. Chem. Soc. 125, 15690-15691 (2003).

15. Jiang, Z. W. et al. Controllable synthesis of porphyrin-based 2D lanthanide metal-organic frameworks with thickness- and metal-node-dependent photocatalytic performance. Angew. Chem. Int. Ed. 59, 3300-3306 (2020).

16. Choi, J. et al. Energy efficient electrochemical reduction of $\mathrm{CO}_{2}$ to $\mathrm{CO}$ using a three-dimensional porphyrin/graphene hydrogel. Energy Environ. Sci. 12, 747-755 (2019).

17. Bengasi, G. et al. Conductive fused porphyrin tapes on sensitive substrates by a chemical vapor deposition approach. Angew. Chem. Int. Ed. 58, 2103-2108 (2019)

18. Tsuda, A. \& Osuka, A. Fully conjugated porphyrin tapes with electronic absorption bands that reach into infrared. Science 293, 79 (2001).

19. Haver, R. et al. Tuning the circumference of six-porphyrin nanorings. J. Am Chem. Soc. 141, 7965-7971 (2019).

20. O'Sullivan, M. C. et al. Vernier templating and synthesis of a 12-porphyrin nano-ring. Nature 469, 72-75 (2011).

21. Higashino, T., Yamada, T., Sakurai, T., Seki, S. \& Imahori, H. Fusing porphyrins and phospholes: synthesis and analysis of a phosphoruscontaining porphyrin. Angew. Chem. Int. Ed. 55, 12311-12315 (2016).

22. Chmielewski, P. J., Latos-Grażyński, L., Rachlewicz, K. \& Glowiak, T. Tetra-ptolylporphyrin with an inverted pyrrole ring: a novel isomer of porphyrin. Angew. Chem. Ed. Engl. 33, 779-781 (1994). 
23. Furuta, H., Asano, T. \& Ogawa, T. "N-confused porphyrin": a new isomer of tetraphenylporphyrin. J. Am. Chem. Soc. 116, 767-768 (1994).

24. Chmielewski, P. J. Synthesis and characterization of a directly linked Nconfused porphyrin dimer. Angew. Chem. 116, 5773-5776 (2004).

25. Ooi, S., Tanaka, T., Park, K. H., Kim, D. \& Osuka, A. Triply linked corrole dimers. Angew. Chem. 128, 6645-6649 (2016).

26. Tsuda, A., Furuta, H. \& Osuka, A. Completely fused diporphyrins and triporphyrin. Angew. Chem. Int. Ed. 39, 2549-2552 (2000).

27. Saito, S. \& Osuka, A. Expanded porphyrins: intriguing structures, electronic properties, and reactivities. Angew. Chem. Int. Ed. 50, 4342-4373 (2011).

28. Li, Q. et al. Regioselectively halogenated expanded porphyrinoids as building blocks for constructing porphyrin-porphyrinoid heterodyads with tunable energy transfer. J. Am. Chem. Soc. 141, 5294-5302 (2019).

29. Wei, P. et al. Neo-fused hexaphyrin: a molecular puzzle containing an Nlinked pentaphyrin. Angew. Chem. Int. Ed. 53, 14069-14073 (2014).

30. Ohimain, E. I. Methanol contamination in traditionally fermented alcoholic beverages: the microbial dimension. Springerplus 5, 1607 (2016).

31. Arslan, M. M. et al. Analysis of methanol and its derivatives in illegally produced alcoholic beverages. J. Forensic Leg. Med. 33, 56-60 (2015).

32. Bilgi, M. \& Ayranci, E. Development of amperometric biosensors using screen-printed carbon electrodes modified with conducting polymer and nanomaterials for the analysis of ethanol, methanol and their mixtures. $J$. Electroanal. Chem. 823, 588-592 (2018).

33. Sharma, K., Sharma, S. P. \& Lahiri, S. Novel method for identification and quantification of methanol and ethanol in alcoholic beverages by gas chromatography-Fourier transform infrared spectroscopy and horizontal attenuated total reflectance Fourier transform infrared spectroscopy. J. AOAC Int. 92, 518-526 (2019).

34. de Paula Pereira, P. A., Sousa Santos, E. T., de Freitas Ferreira, T. \& de Andrade, J. B. Determination of methanol and ethanol by gas chromatrography following air sampling onto florisil cartridges and their concentrations at urban sites in the three largest cities in Brazil. Talanta 49, 245-252 (1999).

35. Yang, Y. R. et al. Determination of methanol in alcoholic beverages by twodimensional near-infrared correlation spectroscopy. Anal. Lett. 49, 2279-2289 (2016).

36. Góes, R. E. D., Fabris, L. V. M., Muller, M. \& Fabris, J. L. Light-assisted detection of methanol in contaminated spirits. J. Lightwave Technol. 34, 4499-4505 (2016).

37. Wen, G., Wen, X., Shuang, S. \& Choi, M. M. F. Whole-cell biosensor for determination of methanol. Sens. Actuators B 201, 586-591 (2014).

38. Harraz, F. A. et al. Conducting polythiophene/ $\alpha-\mathrm{Fe}_{2} \mathrm{O}_{3}$ nanocomposite for efficient methanol electrochemical sensor. Appl. Surf. Sci. 508, 145226 (2020).

39. Kumar, V., Kundu, S., Sk, B. \& Patra, A. A naked-eye colorimetric sensor for methanol and 'turn-on' fluorescence detection of $\mathrm{Al}^{3+}$. New J. Chem. 43, 18582-18589 (2019).

40. Zou, X.-Y. et al. Visual detection of methanol in alcoholic beverages using alcohol-responsive poly( $\mathrm{N}$-isopropylacrylamide-co-N,N-dimethylacrylamide) copolymers as indicators. RSC Adv. 4, 61711-61721 (2014).

41. Zhang, X. \& Chen, Y. A light-modulated chemosensor for methanol with ratiometry and colorimetry. Anal. Chim. Acta 650, 254-257 (2009).

42. Kumar, V., Kumar, A., Diwan, U., Singh, M. K. \& Upadhyay, K. K. A radical approach for fluorescent turn 'on' detection, differentiation and bioimaging of methanol. Org. Biomol. Chem. 13, 8822-8826 (2015).

43. Wu, Z., Fu, X. \& Wang, Y. Click synthesis of a triphenylamine-based fluorescent methanol probe with a unique D- $\pi-A$ structure. Sens. Actuators $B$ 245, 406-413 (2017).

44. Cho, D.-G. \& Sessler, J. L. Modern reaction-based indicator systems. Chem. Soc. Rev. 38, 1647-1662 (2009).

45. Chan, J., Dodani, S. C. \& Chang, C. J. Reaction-based small-molecule fluorescent probes for chemoselective bioimaging. Nat. Chem. 4, 973-984 (2012).

46. Hiroto, S., Miyake, Y. \& Shinokubo, H. Synthesis and functionalization of porphyrins through organometallic methodologies. Chem. Rev. 117, 2910-3043 (2017).

47. Bonifazi, D. et al. Exceptional redox and photophysical properties of a triply fused diporphyrin-C60 conjugate: novel scaffolds for multicharge storage in molecular scale. Electron. Angew. Chem. Int. Ed. 42, 4966-4970 (2003).

48. Mori, H. et al. Meso-meso linked porphyrin-[26]hexaphyrin-porphyrin hybrid arrays and their triply linked tapes exhibiting strong absorption bands in the NIR region. J. Am. Chem. Soc. 137, 2097-2106 (2015).

49. Sheldrick, G. M. SHELXS97 and SHELXL97 Programs for Crystal Structure Solution and Refinement (University of Göttigen, 1997).

50. Hehre, W. J., Ditchfield, R. \& Pople, J. A. Self-consistent molecular orbital methods. XII. Further extensions of Gaussian-type basis sets for use in molecular orbital studies of organic molecules. J. Chem. Phys. 56, 2257-2261 (1972).

51. Blanqui, F., Jouannaud, J.-P. \& Okada, M. Corrigendum to "Inductive-datatype systems" [Theoret. Comput. Sci. 272 (1-2) (2002) 41-68]. Theor. Comput. Sci. 817, 81-82 (2020).
52. Costa, A. C. et al. DFT: B3LYP/6-311G (d, p) vibrational analysis of bis(diethyldithiocarbamate)zinc (II) and natural bond orbitals. Spectrochim. Acta Part A 105, 251-258 (2013)

53. Boese, A. D. \& Martin, J. M. L. Development of density functionals for thermochemical kinetics. J. Chem. Phys. 121, 3405-3416 (2004).

54. Wang, C.-W., Hui, K. \& Chai, J.-D. Short- and long-range corrected hybrid density functionals with the D3 dispersion corrections. J. Chem. Phys. 145 204101 (2016)

55. Kim, T. et al. Exciton coupling dynamics in syn- and anti-type $\beta-\beta$ linked Zn(ii) porphyrin linear arrays. Phys. Chem. Chem. Phys. 18, 23105-23110 (2016).

56. Kim, Y. H. et al. Photophysical properties of long rodlike meso-meso-linked zinc(II) porphyrins investigated by time-resolved laser spectroscopic methods. J. Am. Chem. Soc. 123, 76-86 (2001).

57. Tanaka, T. \& Osuka, A. Conjugated porphyrin arrays: synthesis, properties and applications for functional materials. Chem. Soc. Rev. 44, 943-969 (2015).

58. Becke, A. D. Density-functional exchange-energy approximation with correct asymptotic behavior. Phys. Rev. A 38, 3098-3100 (1988).

59. Adamo, C. \& Barone, V. Toward reliable density functional methods without adjustable parameters: the PBE0 model. J. Chem. Phys. 110, 6158-6170 (1999).

60. Yanai, T., Tew, D. P. \& Handy, N. C. A new hybrid exchange-correlation functional using the Coulomb-attenuating method (CAM-B3LYP). Chem. Phys. Lett. 393, 51-57 (2004).

61. Chai, J.-D. \& Head-Gordon, M. Long-range corrected hybrid density functionals with damped atom-atom dispersion corrections. Phys. Chem. Chem. Phys. 10, 6615-6620 (2008).

62. Zhao, Y. \& Truhlar, D. G. The M06 suite of density functionals for main group thermochemistry, thermochemical kinetics, noncovalent interactions, excited states, and transition elements: two new functionals and systematic testing of four M06-class functionals and 12 other functionals. Theor. Chem. Acc. 120, 215-241 (2008)

63. Uttamlal, M. \& Sheila Holmes-Smith, A. The excitation wavelength dependent fluorescence of porphyrins. Chem. Phys. Lett. 454, 223-228 (2008).

64. de la Torre, G., Giacalone, F., Segura, J. L., Martín, N. \& Guldi, D. M. Electronic communication through $\pi$-conjugated wires in covalently linked porphyrin/C60 ensembles. Chem. Eur. J. 11, 1267-1280 (2005).

65. Drobizhev, M. et al. Understanding strong two-photon absorption in $\pi$ conjugated porphyrin dimers via double-resonance enhancement in a threelevel model. J. Am. Chem. Soc. 126, 15352-15353 (2004).

66. Shortreed, M., Kopelman, R., Kuhn, M. \& Hoyland, B. Fluorescent fiber-optic calcium sensor for physiological measurements. Anal. Chem. 68, 1414-1418 (1996).

67. Dolomanov, O. V., Bourhis, L. J., Gildea, R. J., Howard, J. A. K. \& Puschmann, H. OLEX2: a complete structure solution, refinement and analysis program. J. Appl. Crystallogr. 42, 339-341 (2009).

68. Sheldrick, G. M. Crystal structure refinement with SHELXL. Acta Crystallogr. C71, 3-8 (2015)

69. Tomasi, J., Mennucci, B. \& Cammi, R. Quantum mechanical continuum solvation models. Chem. Rev. 105, 2999-3094 (2005).

\section{Acknowledgements}

This work at ECUST was financially supported by the Shanghai Municipal Science and Technology Major Project (Grant No. 2018SHZDZX03), the International Cooperation Program of Shanghai Science and Technology Committee (17520750100), NSFC (21971063, 21772041, 21702062, 21811530005), Program of Introducing Talents of Discipline to Universities (B160170), the Fundamental Research Funds for the Central Universities (222201717003), Program of Shanghai Academic Research Leader (20XD1401400), China Postdoctoral Science Foundation (2019TQ0092, 2020M671016), and Natural Science Foundation of Shanghai (20ZR1414100). The work in Austin was supported by the National Science Foundation (CHE-1807152 to J.L.S.) and the Robert A. Welch Foundation (F-0018 to J.L.S.). We thank the Research Center of Analysis and Test of East China University of Science and Technology for help with material characterization.

\section{Author contributions}

Y.X. and J.L.S. conceived the project and designed the molecules. Q.L. and C.L. performed the compound syntheses and characterization, grew the single crystals, studied the crystal structures, and measured the absorption spectra. F.S. and X.W. proposed the mechanisms and optimized the synthetic routes leading to all compounds. G.B., C.Z., and H.A. did the theoretical calculations. T.G., X.L., and W.Z. carried out the electrochemical measurements. Y.D. carried out the methanol response measurements. Y.X., J.L.S., and Q.L. wrote the manuscript. All authors discussed about results and contributed to the interpretation of the data.

\section{Competing interests}

The authors declare no competing interests. 


\section{Additional information}

Supplementary information is available for this paper at https://doi.org/10.1038/s41467020-19118-9.

Correspondence and requests for materials should be addressed to J.L.S. or Y.X.

Peer review information Nature Communications thanks the anonymous reviewers for their contribution to the peer review of this work.

Reprints and permission information is available at http://www.nature.com/reprints

Publisher's note Springer Nature remains neutral with regard to jurisdictional claims in published maps and institutional affiliations. (c) (i) Open Access This article is licensed under a Creative Commons Attribution 4.0 International License, which permits use, sharing, adaptation, distribution and reproduction in any medium or format, as long as you give appropriate credit to the original author(s) and the source, provide a link to the Creative Commons license, and indicate if changes were made. The images or other third party material in this article are included in the article's Creative Commons license, unless indicated otherwise in a credit line to the material. If material is not included in the article's Creative Commons license and your intended use is not permitted by statutory regulation or exceeds the permitted use, you will need to obtain permission directly from the copyright holder. To view a copy of this license, visit http://creativecommons.org/ licenses/by/4.0/.

(C) The Author(s) 2020 